\title{
A kinetic model of $\beta$-adrenergic control in cardiac myocytes
}

\author{
Shelley Fong ${ }^{1^{*}}$ and Jeffrey J. Saucerman ${ }^{2}$ \\ ${ }^{1}$ Auckland Bioengineering Institute, The University of Auckland, New Zealand \\ ${ }^{2}$ Biomedical Engineering, The University of Virginia, USA
}

\section{ORIGINAL}

\begin{abstract}
The system of equations and figures presented in Saucerman et al. (2003) are verified and reproduced in this paper's curation effort. These checks are performed in MATLAB. With some parameter additions and modifications, we can reproduce all figures with small mismatches.
\end{abstract}

Keywords: $\quad \beta$-adrenergic, cardiac myocyte, MATLAB

\section{Curated Model Implementation}

http://doi.org/10.36903/physiome.16664248

\section{Primary Publications}

J. J. Saucerman, L. L. Brunton, A. P. Michailova, and A. D. McCulloch. Modeling $\beta$-adrenergic control of cardiac myocyte contractility in silico. Journal of Biological Chemistry, 278(48):47997-48003, 2003.

OPEN ACCESS Reproducible Model Edited by David P. Nickerson

Curated by Weiwei Ai

${ }^{*}$ Corresponding author s.fong@auckland.ac.nz

Submitted 3 Sept 2021

Accepted 23 Sept 2021

Citation

Fong and Saucerman (2021)

A kinetic model of beta-adrenergic control in cardiac myocytes. Physiome. doi: $10.36903 /$ physiome. 16664248

\section{Introduction}

$\beta$-adrenergic signalling within the cardiac myocyte is a well-documented signalling pathway, but few mathematical models exist of the complete system. Saucerman et al. (2003) published such a model of the activation of the signalling cascade from a ligand binding the receptor on the cell surface, to triggering the activation of the beta- 1 adrenergic receptor ( $\beta 1 A R)$, cyclic AMP (CAMP), adenylyl cyclase (AC), protein kinase A (PKA), and phospholamban (PLB). Subsequent downstream effects on ion channel activity are the result, notably through modifying the activity of calcium channels in the regulation of an action potential.

This present work involves the mathematical curation the model. MATLAB (MATLAB, 2017) code was obtained from the first author of Saucerman et al. (2003) in an effort to reproduce the figures of that paper. While there is not an exact correspondence between equations presented in the paper and code provided, the figure trends are still reproduced, and numeric values are matched with some modifications/additions made to parameter values, as detailed in under Model Modifications.

A persistent workspace of the code is available in the Physiome Model Repository, at https: //models.physiomeproject.org/workspace/6fa.

This workspace includes the MATLAB source code for running the model. The corresponding CellML (Cuellar et al., 2003) implementation is available at https://models.physiomeproject.org/e/ $6 \mathrm{f} 6$ which contains the encoding of the mathematics, but does not reproduce the figures due to limited solver capabilities at time of testing. As such, we have elected to do this present curation in MATLAB. 


\section{Model description}

The model is broken into several modules:

- $\beta 1 \mathrm{AR}$ activation

- Gs protein activation

- cAMP metabolism

- PKA activation

- PLB regulation

- L-type calcium channel (LCC) regulation

Once the cell is activated by an agonist isoproterenol (Iso), the collective effects of these modules are studied downstream. This includes changes to ion channel activity towards the production and management of an action potential.

\section{Model Modifications}

\subsection{Mathematical Equations}

These are the same as reported in the supplementary material of Saucerman et al. (2003) with the exception of the L-type calcium channel. This is a reduced-state version of the original model by Jafri et al. (1998), which was implemented in the MATLAB code provided by the original author. The version of the model published in Saucerman et al. (2003) showed a complete version of the model as in Jafri et al. (1998), replicated to represent phosphorylated states.

\subsection{Parameter Values}

The baseline parameter values are provided in the supplementary material of Saucerman et al. (2003) and in the MATLAB code provided by Saucerman. Missing values on cell geometry are provided in Table 1 which are used in all Figure reproductions.

\begin{tabular}{|l|l|l|}
\hline Name & Value & Source \\
\hline Myocyte volume & $36.8 \mathrm{pL}$ & Bers (2001) \\
Myocyte density & $1.06 \mathrm{~g} / \mathrm{mL}$ & Mendez et al. (1960) \\
\hline
\end{tabular}

Table 1. Parameters sourced for the model, which were not included in the primary paper.

It was found that the density of myocytes needed to increase by 6.4 times in order to better match the primary paper figures.

Experimental conditions specific to each Figure are given in Table 2. For Figures $2 \mathrm{f}-\mathrm{h}$ and $6 \mathrm{~b}$, patch clamp protocols are defined in Table 3.

This system has 44 dependent variables. Unless stated otherwise, all figure reproductions are subject to the same initial conditions (see source code). These values are embedded in the script main.m.

\section{Computational Simulation}

The system of differential-algebraic equations is solved in MATLAB using stiff solver ode15s (Shampine and Reichelt, 1997) with automatic/adaptive time stepping, Re/Tol $=1 e-6$, MaxStep $=$ $5 e-3$. No further packages are required; any machine with the full ODE suite (which includes ode15s solver) should be able to run all simulations.

Run the script main.m. The user will be prompted to specify the desired figure reproduction: input the appropriate string of format $i a$ where $i$ is the figure number and $a$ is the subfigure letter: for example, $2 b$. 


\begin{tabular}{|c|c|c|c|}
\hline Figure & Parameter values & Number of iterations & $\mathrm{T}(\mathrm{min})$ \\
\hline $2 a$ & & 1 & 18 \\
\hline $2 b$ & $\begin{array}{l}{\left[\text { Iso }^{\dagger}\right]=\text { logspace }(-3,0.45,8) \mu M} \\
{[I B M X]=100 \mu M}\end{array}$ & 8 & 6 \\
\hline $2 c, 2 d$ & $\begin{array}{l}{[\text { Iso }]=1 \mu M} \\
{[[P K I]=\{0,0.052\} \mu M} \\
{\left[c A M P^{\dagger}\right]=\operatorname{logspace}(-1.9,2,6) \mu M}\end{array}$ & 6 & 1 \\
\hline $2 e$ & {$\left[\right.$ Iso $\left.^{\dagger}\right]=\operatorname{logspace}(-6,0,20) \mu M$} & 20 & 30 \\
\hline $2 f$ & {$\left[I s 0^{\dagger}\right]=\{0,1\} \mu M$} & 2 & 1 \\
\hline $2 \mathrm{~g}, 2 \mathrm{~h}$ & {$\left[I s 0^{\dagger}\right]=\{0,1\} \mu M$} & 2 & 1 \\
\hline $3 a$ & $\begin{array}{l}{\left[I s 0^{\dagger}\right]=\text { logspace }(-3,0,8) \mu M} \\
{[A C]_{\text {tot }} *=3} \\
{\left[\beta 1 A R_{\text {tot }}\right] *=3} \\
K_{\text {Gs }} /=3\end{array}$ & 32 & 7 \\
\hline $3 b$ & $\begin{array}{l}{\left[I s 0^{\dagger}\right]=\text { logspace }(-3,0,8) \mu \mathrm{M}} \\
{[A C]_{\text {tot }} *=1.5} \\
{\left[\beta 1 A R_{\text {tot }}\right] *=1.8} \\
K_{\text {Gs }} /=1.9\end{array}$ & 32 & 7 \\
\hline $4 a, 4 b$ & $\begin{array}{l}{[I B M X]=250 \mu M} \\
{\left[I s o^{\dagger}\right]=\{1,0,1\} \mu M} \\
k_{\text {hyd }}^{\dagger} *=\{1,0.05,0.05\}\end{array}$ & 3 & 3 \\
\hline 5 & $\begin{array}{l}{\left[I s o^{\dagger}\right]=\operatorname{logspace}(-4,0,8) \mu M} \\
{\left[P K A_{I, t o t}\right] *=0.05} \\
{\left[P K I_{\text {tot }}\right] *=0.05}\end{array}$ & 24 & 20 \\
\hline $6 a$ & $\begin{array}{l}{\left[I s o^{\dagger}\right]=\{1,1,1\} \mu M} \\
{\left[P L B p^{\dagger}\right]^{t=0} *=\{1,0,1\}} \\
{\left[L C C a p^{\dagger}\right]^{t=0} *=\{1,0,1\}} \\
{\left[L C C b p^{\dagger}\right]^{t=0} *=\{1,0,1\}}\end{array}$ & 3 & 1 \\
\hline $6 b$ & $\begin{array}{l}{\left[I^{\prime} o^{\dagger}\right]=\{0,1,0,1\} \mu M} \\
\text { fracPLB }^{\dagger} *=\{1,1,0,0\} \\
\text { fracPLBo }^{\dagger} *=\{1,1,0,0\}\end{array}$ & 4 & 1 \\
\hline
\end{tabular}

Table 2. Specific protocol followed in the reproduction of each figure, run for a span of $T$ minutes. $*=, /=$ indicate multiplication or division of parameter by the specified value respectively. $\uparrow$ denotes the variable value being changed with every iteration. One iteration constitutes running the entire model once. For multiple species being changed for one figure: for the $n$th loop iteration, the value of the species is given by the $n$th value in the sequence given in the sequence (Column 2) for all marked species. (3a, 3b): four separate iterations for every [Iso]: one control, one with changed $\left[A C_{t o t}\right]$ only, one with changed $\left[\beta 1 A R_{\text {tot }}\right]$ only, and one with change $K_{G s \alpha}$ only. (5): three separate iterations for every [Iso]: one control, one with changed $\left[P K A_{I, t o t}\right]$ only, one with changed $\left[P K I_{\text {tot }}\right]$ only. patch clamping: Figures $2 \mathrm{~g}-\mathrm{h}, 6 \mathrm{~b}$ follow Protocol 1; Figure 2 f follows Protocol 2 (see Table 3).

See primary paper or source code for an explanation of all chemical abbreviations. 


\begin{tabular}{|l|l|}
\hline Protocol & Details \\
\hline 1 & $I_{\text {app }}= \begin{cases}10 \frac{\mu A}{\mu F} & \text { if } \bmod (t+0.9,1)<=5 e-3 \\
0 \frac{\mu A}{\mu F} & \text { otherwise }\end{cases}$ \\
\hline 2 & $V_{c l a m p}= \begin{cases}-10 m V & \text { if } 59.1<t<59.5 \\
-55 m V & \text { otherwise }\end{cases}$ \\
& where $I_{\text {app }}=\frac{V_{\text {clamp }}-V_{m}}{R_{c l a m p}}$
\end{tabular}

Table 3. Patch clamp protocols. $R_{\text {clamp }}=0.02 \Omega$

\begin{tabular}{|c|c|c|c|}
\hline Figure & Species & Original value & Value modified for MATLAB \\
\hline $2 \mathrm{~b}$ & $\max ([\mathrm{Iso}])$ & $10 \mu M$ & $2.8 \mu \mathrm{M}$ \\
\hline $2 c / d$ & [PKI] & $60 n M$ & $52 n M$ \\
\hline $2 f$ & Patch clamp voltage & holding $-80 \mathrm{mV}$, step $0 \mathrm{mV}$ & holding $-55 m V$, step $-10 m V$ \\
\hline $4 a$ & {$[\mathrm{IBMX}]$} & $1 \mathrm{mM}$ & $250 \mu M$ \\
\hline $4 b$ & $\max ([$ Iso] $)$ & $10 \mu M$ & $1 \mu M$ \\
\hline 5 & $\mathrm{PKAl}_{t o t}$ & 0 & $5 \%$ of baseline \\
\hline & $\mathrm{PKI}_{\text {tot }}$ & 0 & $5 \%$ of baseline \\
\hline $6 a$ & [Iso] & - & $1 \mu M$ \\
\hline $6 \mathrm{~b}$ & [lso] & - & $1 \mu M$ \\
\hline & fracPLB & - & 0 \\
\hline
\end{tabular}

Table 4. Modifications made to stated values of primary paper, in the reproduction of the figures.

Figures are highly recommended to be reproduced using parallel computing (parpool, $>=4$ workers) in MATLAB due to the size and stiffness of the code. By default, this is already set up in the script. Otherwise, computations will take up to 30 minutes per figure, on a $2.8 \mathrm{Ghz} / 4$ core machine running on Windows 10 . Once running, keep parpool open (change parallel preferences: adjust time to automatically shut down pool after appropriate amount of time) such that time is not lost waiting for the pool to start up on every simulation.

The original implementation of the model was performed using Berkeley-Madonna software, with a different ODE solver to MATLAB Saucerman et al. (2003). As some simulation values stated in the primary paper resulted in the model being unsolvable in the MATLAB routine, we had to make adjustments as outlined in Table 4.

\section{Model Results}

We employ Engauge Digitizer software (Mitchell et al., 2020) to extract data from Figures of Saucerman et al. (2003) to present alongside results of the present work.

The reproduction of all figures of Saucerman et al. (2003) are given in Figures 1-5, which show good agreement against the data of the primary paper. Solid lines show the output from this curation effort, and crosses show discrete points found by the Engauge Digitizer of the figures originally published.

\section{Discussion}

We demonstrate that figures of Saucerman et al. (2003) can be reproduced given some additions (Table 1) and adjustments (Table 4) made to model parameter values. There are slight deviations 

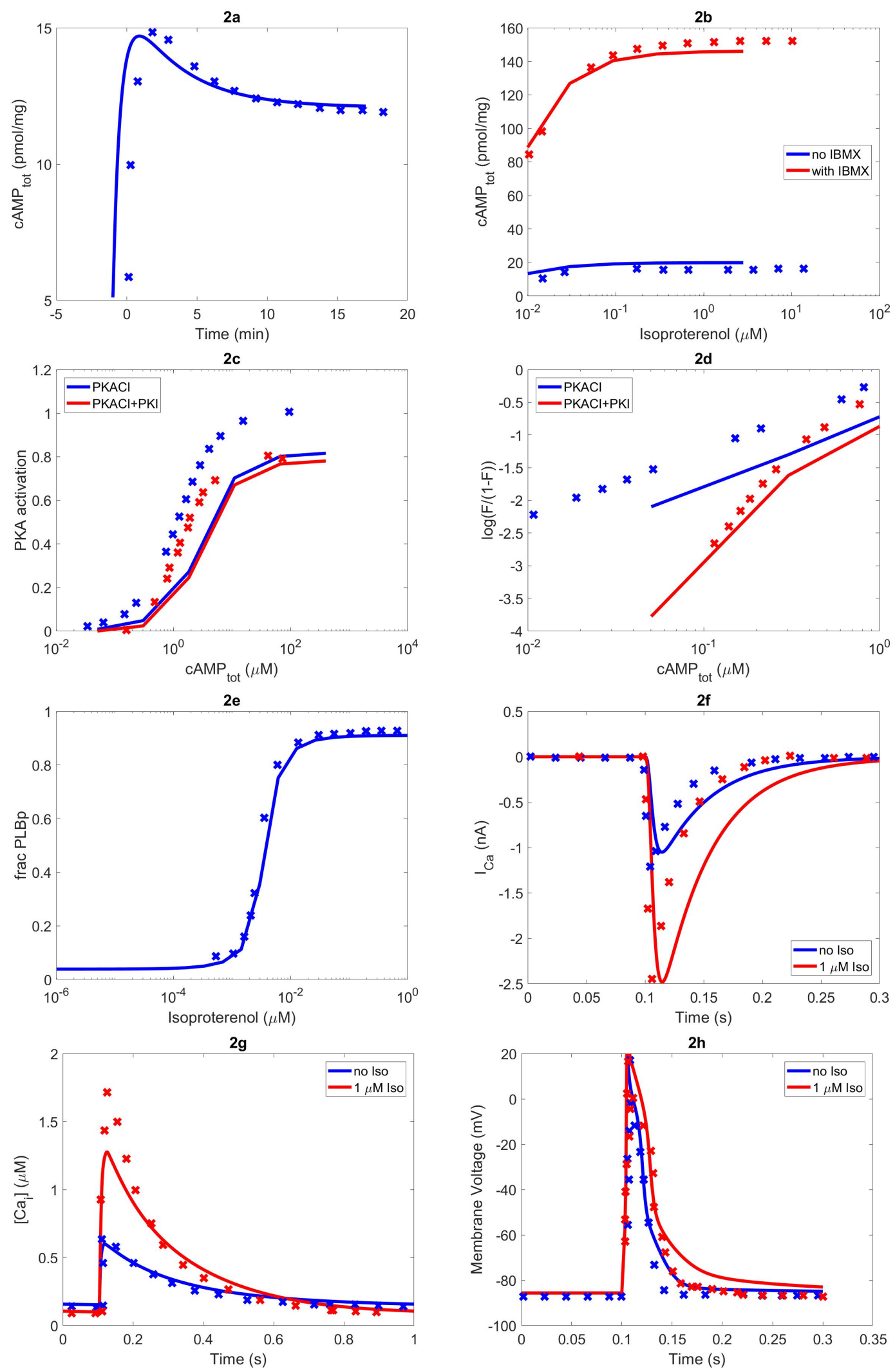

Figure 1. The primary data $(x)$ of Figure 2 with our reproduction of all subfigures $(-)$. Note: (2c) $P K A$ activation $=\frac{[P K A C I]}{2 *\left[P K A I_{\text {tot }}\right]}$ 

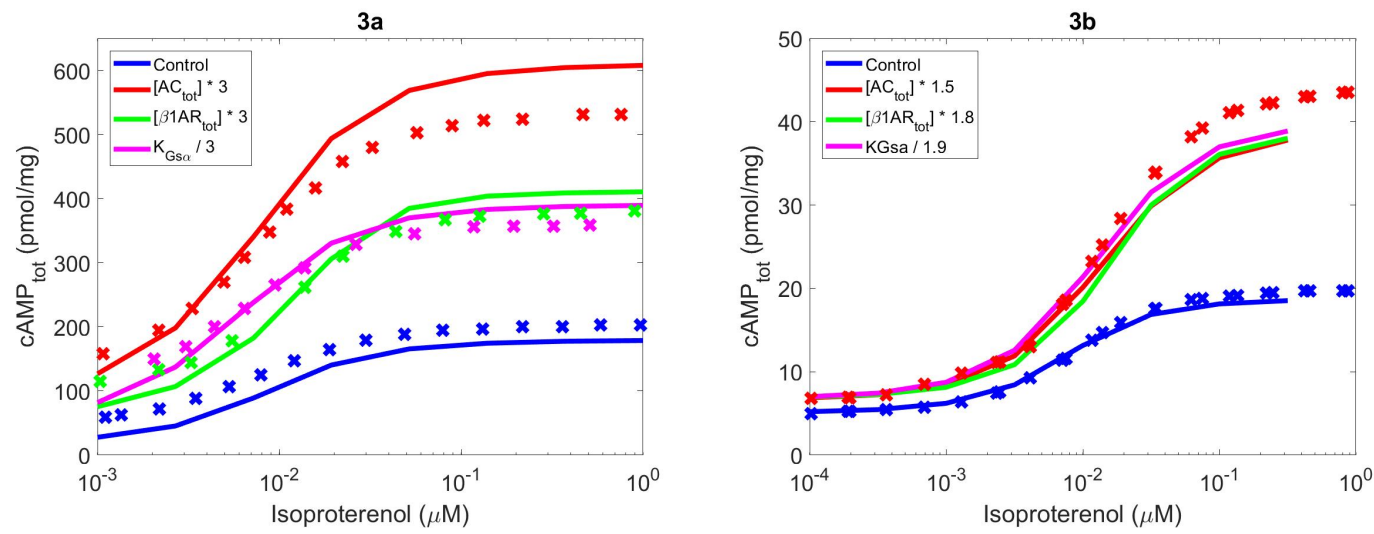

Figure 2. The primary data $(x)$ of Figure 3 with our reproduction of all subfigures $(-)$. Note: lines in (3b) showing modifications to $[A C]_{t o t}, \beta 1 A R, K_{G s \alpha}$ have been grouped into an average 'augmentation' line.
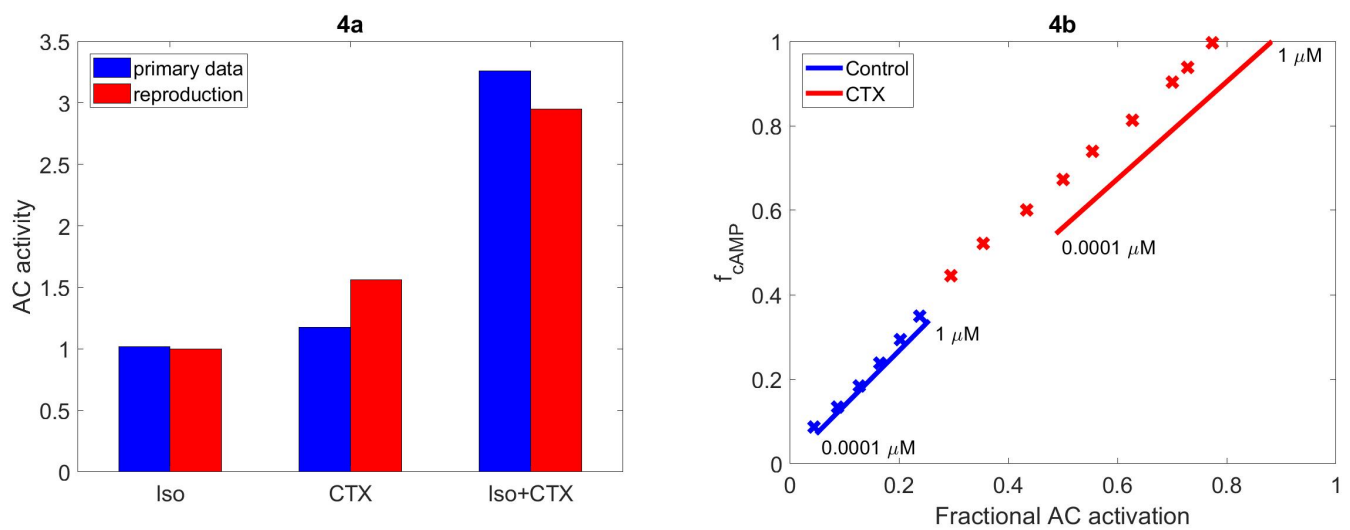

Figure 3. The primary data $(x)$ of Figure 4 with our reproduction of all subfigures $(-)$. (4a): primary data shown in blue, reproduction in red. $A C$ activity $=c A M P_{\text {tot }} .(4 \mathrm{~b})$ : $f_{C A M P}=\frac{c A M P_{t o t}}{C A M P_{t o t, m a x}}$. Annotated numbers on the graph refer to [Iso] set for that experiment.

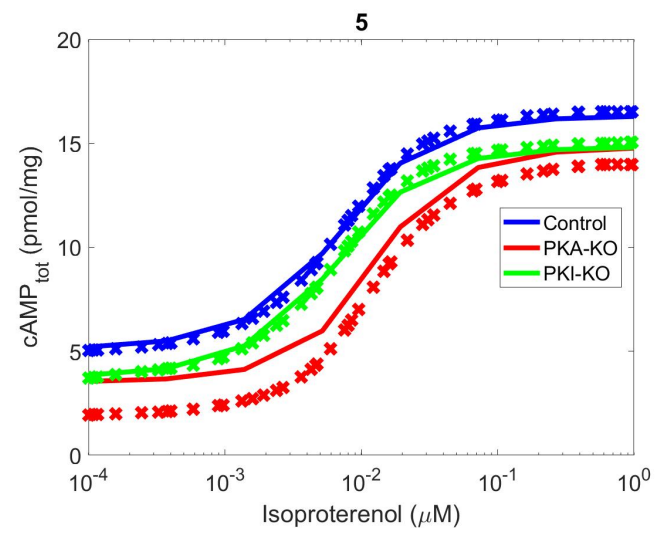

Figure 4. The primary data $(x)$ of Figure 5 with our reproduction $(-)$. 

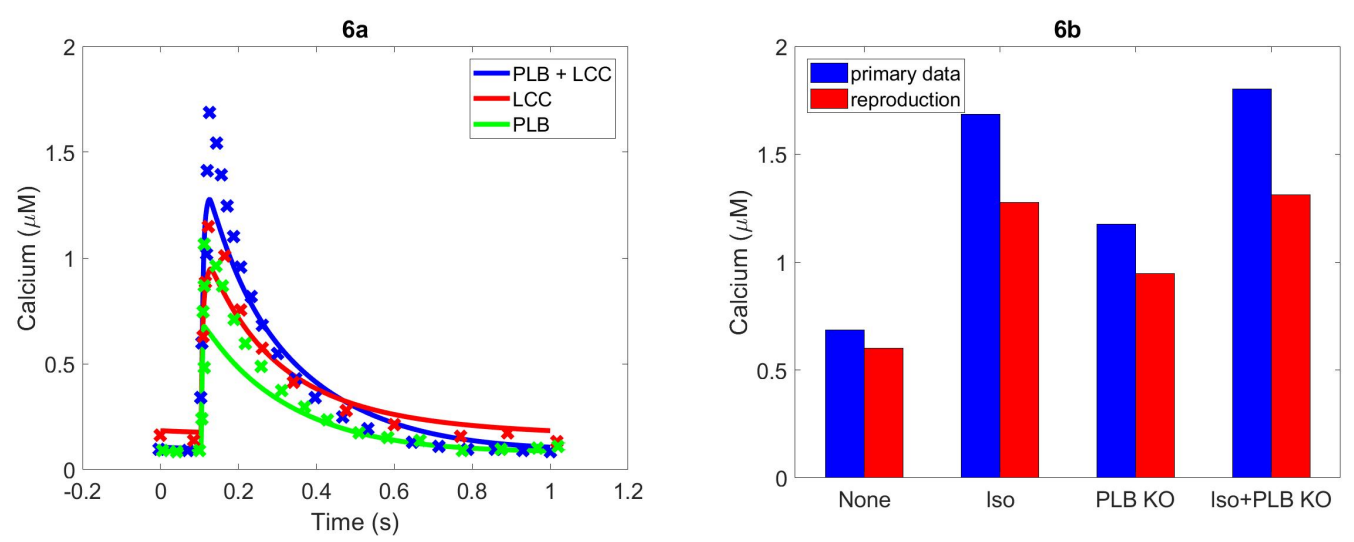

Figure 5. (Left) The primary data $(x)$ of Figure 6a with our reproduction of all subfigures $(-)$. (Right) Primary data shown in blue, reproduction in red.

of the curated model output from the original data which can be attributed to parameter modifications we had to make to ensure a simulation runs to completion (see Figures 1c, 1d, 3a, 3b). Other mismatches may be owing to differences from the original implementation in Berkeley-Madonna, which had different solver settings to this effort. The original code is no longer available.

As this paper follows the tenets of FAIR (Wilkinson et al., 2016), further credibility is lent to the original model. The model has already proven to be valuable to the mathematical modelling community in understanding the workings of $\beta$-adrenergic receptor signalling within cardiac myocytes.

\section{References}

D. Bers. Excitation-contraction coupling and cardiac contractile force, volume 237. Springer Science \& Business Media, 2001.

A. A. Cuellar, C. M. Lloyd, P. F. Nielsen, D. P. Bullivant, D. P. Nickerson, and P. J. Hunter. An overview of cellml 1.1, a biological model description language. SIMULATION, 79(12):740-747, 2003. doi: 10.1177/0037549703040939. URL https://doi.org/10.1177/0037549703040939.

M. S. Jafri, J. J. Rice, and R. L. Winslow. Cardiac ca2+ dynamics: the roles of ryanodine receptor adaptation and sarcoplasmic reticulum load. Biophysical journal, 74(3):1149-1168, 1998.

MATLAB. version 9.3.0713579 (R2017b). The MathWorks Inc., Natick, Massachusetts, 2017.

J. Mendez, A. Keys, J. Anderson, F. Grande, et al. Density of fat and bone mineral of the mammalian body. Metabolism, 9:472-477, 1960.

M. Mitchell, B. Muftakhidinov, T. Winchen, A. Wilms, B. van Schaik, badshah400, Mo-Gul, T. G. Badger, Z. Jędrzejewski-Szmek, kensington, and kylesower. markummitchell/engauge-digitizer: Nonrelease, July 2020. URL https://doi.org/10.5281/zenodo.3941227.

J. J. Saucerman, L. L. Brunton, A. P. Michailova, and A. D. McCulloch. Modeling $\beta$-adrenergic control of cardiac myocyte contractility in silico. Journal of Biological Chemistry, 278(48):47997-48003, 2003.

L. F. Shampine and M. W. Reichelt. The matlab ode suite. SIAM journal on scientific computing, 18 (1):1-22, 1997.

M. D. Wilkinson, M. Dumontier, I. J. Aalbersberg, G. Appleton, M. Axton, A. Baak, N. Blomberg, J.-W. Boiten, L. B. da Silva Santos, P. E. Bourne, et al. The fair guiding principles for scientific data management and stewardship. Scientific data, 3(1):1-9, 2016. 
Reproducibility report for: A kinetic model of beta-adrenergic control in cardiac myocytes.

Submitted to: Physiome

Manuscript number/identifier: S000012

Curation outcome summary: Successfully reproduced all the figures presented in this manuscript.

Box 1: Criteria for repeatability and reproducibility

\section{Model source code provided:}

Source code: a standard procedural language is used (e.g. MATLAB, Python, C)

There are details/documentation on how the source code was compiled

There are details on how to run the code in the provided documentation

$\square$ The initial conditions are provided for each of the simulations

Details for creating reported graphical results from the simulation results

Source code: a declarative language is used (e.g. SBML, CellML, NeuroML)

$\square$ The algorithms used are defined or cited in previous articles

$\square$ The algorithm parameters are defined

$\square$ Post-processing of the results are described in sufficient detail

\section{Executable model provided:}

$\square$ The model is executable without source (e.g. desktop application, compiled code, online service)

$\square$ There are sufficient details to repeat the required simulation experiments

\section{The model is described mathematically in the article(s):}

Equations representing the biological system

There are tables or lists of parameter values

There are tables or lists of initial conditions

Machine-readable tables of parameter values

Machine-readable tables of initial conditions

The simulation experiments using the model are described mathematically in the article:

$\square$ Integration algorithms used are defined

$\square$ Stochastic algorithms used are defined

$\square$ Random number generator algorithms used are defined

$\square$ Parameter fitting algorithms are defined

The paper indicates how the algorithms yield the desired output 
Box 2: Criteria for accessibility

Model/source code is available at a public repository or researcher's web site

$\square$ Prohibitive license provided

$\square$ Permissive license provided

Open-source license provided

All initial conditions and parameters are provided

All simulation experiments are fully defined (events listed, collection times and measurements specified, algorithms provided, simulator specified, etc.)

Box 3: Rules for Credible practice of Modeling and Simulation ${ }^{a}$

aModel credibility is assessed using the Interagency Modeling and Ananlysis Group conformance rubric: https://www.imagwiki.nibib.nih.gov/content/10-simple-rules-conformance-rubric

Define context clearly: Extensive

Use appropriate data: Extensive

Evaluate within context: Extensive

List limitations explicitly: Insufficient

Use version control: Extensive

Document adequately: Extensive

Conform to standards: Insufficient

Box 4: Evaluation

Model and its simulations could be repeated using provided declarative or procedural code

Model and its simulations could be reproduced 
Summary comments: Model and source code are available in the associated OMEX archive. This was used in our attempt to reproduce the results presented in the paper. We successfully ran the scripts provided to reproduce Figure 1 - Figure 5 as presented in this manuscript.

\author{
Anad Raypadeas \\ Anand K. Rampadarath ${ }^{1}$, PhD \\ Curator \\ Center for Reproducible Biomedical Modeling
}

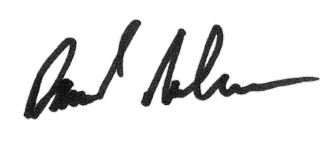

David P. Nickerson, PhD

Curation Service Director

Center for Reproducible Biomedical Modeling

Auckland Bioengineering Institute, University of Auckland

\footnotetext{
1Email: a.rampadarath@auckland.ac.nz
} 